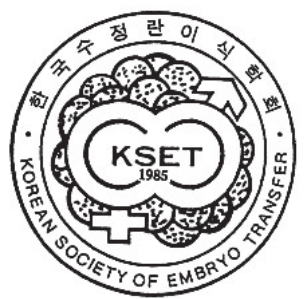

\title{
Influence of Autophagy Induction after Hormone Treatment on Oocytes Maturation of Porcine
}

Sang Hwan Kim ${ }^{1}$ and Jong Taek Yoon ${ }^{1,2, \uparrow^{*}}$

${ }^{1}$ Institute of Genetic Engineering, Hankyong National University, Anseoung, Gyeonggi-do 456-749, Korea

${ }^{2}$ Department of Animal Life Science, Hankyong National University, Anseoung, Gyeonggi-do 456-749, Korea

\section{Abstract}

Here, we evaluated the mode of programmed cell death during porcine oocyte maturation by comparing the two major pathways associated with programmed cell death, apoptosis (type I), and autophagy (type II). We investigated the expression and localization of major genes involved in autophagy and apoptosis at mRNA and protein levels. Furthermore, the effect of hormonal stimulation on autophagy and apoptosis was analyzed. We found that the activity of autophagy-associated genes was increased in the cumulus-oocyte complexes (COCs) following follicle-stimulating hormone (FSH) treatment, while the addition of luteinizing hormone $(\mathrm{LH})$ reversed this effect. The expression of proteins associated with autophagy was the highest in FSH-treated COCs. On the other hand, caspase-3 protein level was maximum in COCs cultured with LH. The treatment with rapamycin resulted in the effect similar to that observed with FSH treatment and increased autophagy activity. Thus, hormonal stimulation of pig oocytes resulted in distinct patterns of maturation. The high-quality oocytes majorly relied on the type II pathway (autophagy), while the type I pathway (apoptosis) was more prominent among poor-quality oocytes. Further investigation of this distinction may allow the development of techniques to produce high-quality oocytes in porcine in vitro fertilization.

Received : 28 November 2018

Revised : 13 December 2018

Accepted : 13 December 2018

Key Words : Apoptosis, Autophagy, FSH, LH, Maturation oocyte, Porcine

\footnotetext{
* The authors contributed equally to this work

+ Correspondence: Jong Taek Yoon (ORCID: 0000-0003-0996-6912) Tel: +82-31-670-5255, +82-31-670-5094, Fax: +82-31-675-8265,

E-mail address: jtyoon@hknu.ac.kr
} 


\section{INTRODUCTION}

Oocyte maturation is arguably an important step in high-quality blastocyst in vitro fertilization (IVF) systems. Collection of immature oocytes and their subsequent in vitro maturation (IVM) perturb oocyte physiology and result in oocytes with reduced developmental competence as compared with those matured in vivo under natural condition. Offsprings have been produced using oocyte IVM, IVF, and in vitro culture of resulting embryos (Brackett et al., 1982; Eyestone and First, 1989; Yoshida et al., 1993; Kikuchi et al., 1999). However, only a small percentage of the original pool of immature oocytes placed into an IVM system is capable of developing into the blastocyst stage and subsequently resulting in pregnancy. Immature oocytes may undergo initiation and completion of nuclear maturation in vitro and sometimes be fertilized; however, these oocytes are often incapable of supporting the subsequent embryonic development (Edwards et al., 1969; Cohen et al., 1980; Tilly, 2001). Although meiosis or nuclear maturation may be completed successfully in vitro, a variety of other processes occurring within the oocyte cytoplasm are necessary to complete developmental competence following fertilization. The successful completion of these events is independent of nuclear maturation and these events are collectively referred to as "cytoplasmic maturation" (Eppig, 1996; Eppig et al., 1996). Cytoplasmic maturation involves cellular processes such as accumulation of mRNAs and proteins, reorganization of the cytoskeleton and organelles, and changes in the cellular metabolism. In addition, cumulus-oocyte complexes (COCs) to matured is the result of growth of the oocyte, and subsequent nuclear and cytoplasmic maturation. During this maturation time, the information necessary for early development is transcribed and stored in the form of RNA or translated and stored in the form of protein. Following germinal vesicle breakdown, transcription ceases and nuclear maturation progresses until the cell cycle arrest at metaphase II of meiosis. As nuclear maturation rates are often high ( $\geq 70 \%$ ), the deficient development of in vitro matured oocytes is likely associated with inadequate cytoplasmic maturation. Incomplete cytoplasmic maturation in pigs is manifested as a decrease in the ability of the fertilized in vitro matured oocytes to develop into the blastocyst stage (Singh et al., 1997). Furthermore, appropriate oocyte maturation is important for successful fertilization and subsequent embryo development. So far, little is known about the apoptosis mechanisms involved in the maturation process of the pig eggs. According to a study, cumulus cell in accordance with the maturity of immature eggs and oocytes for the reconstruction of cells through the reconstruction of MMPs (Metrix metalloproteinase) determine the maturity of oocytes which is known. Has suggested that they can. In human oocytes, the change in the cytoplasm of the cumulus cell is related to apoptosis and has a significant impact on oocytes maturity. Our results show that during oocyte maturation, distinct types of programmed cell death (PCD: apoptosis and autophagy) are expressed in the cumulus cell and cytoplasm of ovum. We believe that the differential pattern of PCD type and hormones during ovum maturation may provide an insight into the effects of PCD mechanism of development of cumulus cells and oocyte cytoplasm.

\section{MATERIALS AND METHODS}

\section{Collection and preparation of ovarian tissue}

Pig ovaries were collected from gilts at a local slaughterhouse and transported within $2 \mathrm{~h}$ to the laboratory in preincubated saline solution supplemented with $100 \mathrm{IU} / \mathrm{mL}$ penicillin $\mathrm{G}$ and $100 \mu \mathrm{g} / \mathrm{mL}$ streptomycin at $30-35^{\circ} \mathrm{C}$. The follicular fluid from follicles ( 3 to $6 \mathrm{~mm}$ in diameter) was aspirated using an 18 gauge needle attached to a $5 \mathrm{~mL}$ disposable syringe. The fluids were collected in a $15 \mathrm{~mL}$ round-bottom tube (SPL, Seoul, Korea) and incubated for $10 \mathrm{~min}$. With the help of a dissecting microscope, all naked oocytes, COCs, and debris were removed from the aspirate and washed twice in phosphate-buffered saline (PBS). Grade 1 oocytes (oocytes with cumulus cells and good cytoplasm) in COCs were collected and placed under a microscope after being washed and resuspended in HEPES-buffered TCM-199 (Gibco, Rockville, MD, USA) supplemented with $10 \mu \mathrm{l} / \mathrm{mL}$ antibiotic - antimycotic (Gibco, Rockville, MD, USA) and $0.3 \%(\mathrm{w} / \mathrm{v})$ bovine serum albumin (BSA; Sigma, St. Louis, MO, USA). Experiments were conducted following the recommendations specified in the Guide for the Care and Use of Laboratory Animals issued by the National Institutes of Health. The protocol was approved by the Committee on the Ethics of Animal Experiments of Hankyong National University (Permit Number: 2013-1).

\section{In-vitro maturation of pig oocytes with hormone treatment medium}

The oocytes were suspended in TCM-199 and washed once with preincubated IVM medium. We checked the affinity of final oocytes and the binding ability of cumulus cells. These 
were transferred into about 500ul IVM medium in a four-well dish (25 to 38 oocytes in each well) and matured in vitro at $39{ }^{\circ} \mathrm{C}$ in a $5 \%$ carbon dioxide $(\mathrm{CO} 2)$ incubator. In the one-step method, oocytes were matured in vitro in fresh TCM-199 supplemented with 10\% FBS and hormones (follicle-stimulating hormone [FSH], luteinizing hormone [LH], and $\mathrm{FSH}+\mathrm{LH})$. In the second method, fresh TCM-199 supplemented with $10 \%$ FBS (normal group medium) was used and 100nM rapamycin solution was added to each well, followed by incubation for 44 $\mathrm{h}$ at $38{ }^{\circ} \mathrm{C}$ in a humidified atmosphere containing 5\% CO2 and 95\% air. The composition of media used in this experiment is shown in Table 1. Oocyte maturity was evaluated after IVM for $44 \mathrm{~h}$ without medium change. The matured oocyte and media were saved for real-time polymerase chain reaction (PCR), western blot analysis, and immunofluorescence assays.

\section{Quantitative reverse transcription (RT)-PCR analysis}

Total RNA was extracted from the matured oocytes (COCs; About 100 oocytes were used.) using TRIzol reagent (Invitrogen, CA, USA) and treated with DNAse (Ambion, Austin, TX), as per the manufacturer's instruction. The ratio of A260:A280 was $>1.8$ for all RNA samples. RNA integrity was assessed by examining the $28 \mathrm{~S}$ and $18 \mathrm{~S}$ ribosomal RNA bands on ethidium bromide-stained agarose formaldehyde gels. The samples were stored at $-80{ }^{\circ} \mathrm{C}$ until use. First-strand cDNA was synthesized by reverse transcription of mRNA using Oligo(dT) primer and SuperScript II Reverse Transcriptase (Invitrogen, Grand Island, NY). For real-time RT-PCR, Line-gene K (Bioer Technology, Tokyo, Japan) was used to make a final reaction volume of $25 \mathrm{ul}$ with SYBR Green (TOYOBO, Osaka, Japan). The method of Rotor-Gene Real-Time Software 6.0 was used to analyze the cycle threshold $(\mathrm{Ct})$ as well as to obtain a semi-log amplification plot. The relative expression level of each gene was calculated using the $2-\Delta \Delta \mathrm{Ct}$ method by normalization with porcine glyceraldehyde-3-phosphate dehydrogenase (GAPDH) mRNA levels. The primers listed in Table 2. were used for PCR.

\section{Enzyme-linked immunosorbent assay (ELISA) analysis of survival-related genes in each treatment group}

For the analysis of survival-related gene expression, each COC

Table 1. Composition of the in vitro maturation medium

\begin{tabular}{ccccccccc}
\hline & & \multicolumn{3}{c}{ IVM treatment } & medium & & \\
& OCNor & OCF & OCL & OCFL & ROCNor & ROCF & ROCL & ROCFL \\
TCM-199 $(\mu \mathrm{L})$ & 425 & 425 & 425 & 425 & 425 & 425 & 425 & 425 \\
FBS $(\%)$ & 10 & 10 & 10 & 10 & 10 & 10 & 10 & 10 \\
E2 $(\mu \mathrm{g} / \mathrm{mL})$ & 0.5 & 0.5 & 0.5 & 0.5 & 0.5 & 0.5 & 0.5 & 0.5 \\
Rapamycin $(\mathrm{nM})$ & - & - & - & - & 100 & 100 & 100 & 100 \\
EGF $(\mathrm{ng} / \mathrm{mL})$ & 30 & 30 & 30 & 30 & 30 & 30 & 30 & 30 \\
FSH $(\mathrm{IU})$ & - & 10 & - & 5 & - & 10 & - & 5 \\
LH $(\mu \mathrm{g} / \mathrm{mL})$ & - & - & 10 & 5 & - & - & 10 \\
\hline
\end{tabular}

Table 2. Primers for real-time PCR analysis of autophagy and apoptosis-associated genes

\begin{tabular}{|c|c|c|}
\hline Primer & Sequence & $\begin{array}{l}\text { Product size } \\
\text { (base pair) }\end{array}$ \\
\hline Porcine GAPDH for & 5'-CCCGTTCGACAGACAGCCGTG-3' & \multirow{2}{*}{238} \\
\hline Porcine GAPDH rev & 5'-CCGCCTTGACTGTGCCGTGG-3' & \\
\hline PorcineMAP1LC3A for & 5'-AGAAGCAGCTGCCAGTCCTGGACA-3' & \multirow{2}{*}{687} \\
\hline Porcine MAP1LC3A rev & 5'-CAGGCAGGCCTGAGCAATCTTTATT-3' & \\
\hline Porcine ATG5 for & 5'-AGAGAAGTCTGTCCTTCCGCAGTCG-3' & \multirow{2}{*}{241} \\
\hline Porcine ATG5 rev & 5'-AAGCAGAAGGGTGACATGCTCTGGT-3' & \\
\hline Porcine 20a-HSD for & 5' GCCATTGCCAAAAAGCACAAG 3' & \multirow{2}{*}{210} \\
\hline Porcine 20a-HSD rev & 5' GGAAAGCGGATAGTCAGGGTGATC & \\
\hline Porcine Casp-3 for & 5'-CATGGTCAGGCCTTGTGAAGCTGAC-3' & \multirow{2}{*}{150} \\
\hline Porcine Casp-3 rev & 5'-TCTTCTTCATGACCTCACCGTCGGG-3' & \\
\hline
\end{tabular}


protein was coated at $4{ }^{\circ} \mathrm{C}$ for 1 day with primary antibodies against mammalian target of rapamycin (mTOR) in a 96-well ELISA plate. The plate was washed twice with washing buffer $(1 \times$ PBS with $2.5 \%$ Triton X-100) and blocked using $1 \%$ skim milk blocking solution at $4{ }^{\circ} \mathrm{C}$ for $24 \mathrm{~h}$. After washing with the washing buffer, the protein activity was detected using a secondary antibody and a substrate solution (R\&D Systems, USA). The reaction was stopped with the addition of $1 \mathrm{M}$ $\mathrm{NH} 2 \mathrm{SO} 4$ and absorbance was measured at $450 \mathrm{~nm}$ wavelength. The levels of proteins were determined according to a standard curve, which considered four parameters based on the following equation: $\mathrm{y}=(\mathrm{A} 2 \mathrm{D}) /(1+[\mathrm{x} / \mathrm{C}] \wedge \mathrm{B}])+\mathrm{D}$.

\section{Western blot analysis}

Protein extracts from COCs were separated in duplicates by sodium dodecyl sulfate polyacrylamide gel electrophoresis (SDS-PAGE) using 13\% gels and transferred onto polyvinylidene fluoride (PVDF) membranes (Bio-Rad, Hercules, CA). The membranes were blocked with $5 \%$ non-fat dry milk overnight at $4{ }^{\circ} \mathrm{C}$ and washed for $10 \mathrm{~min}$ with the washing buffer (TBS-T; $0.1 \% \mathrm{v} / \mathrm{v}$ Tween-20, $50 \mathrm{mM}$ Tris-HCl, $\mathrm{pH}$ 7.6, and $200 \mathrm{mM}$ sodium chloride $[\mathrm{NaCl}])$. The membranes were incubated for 2 $\mathrm{h}$ with primary antibodies (1:1,000 dilution) against the active form of 20-a-hydroxysteroid dehydrogenase (20a-HSD; Kim 2014 et al., Ansung, Korea), caspase-3 (ab4051, Abcam, San Francisco, USA), autophagy related 5 (ATG5; sc-8667, Santa Cruz Biotechnology Inc., Texas, USA), microtubule-associated protein 1 light chain 3 alpha (MAP1LC3A; sc-134226, Santa Cruz Biotechnology Inc., Texas, USA), and $\beta$-actin (sc-47778, Santa Cruz Biotechnology Inc., Texas, USA) at 1:1000 dilutions. The primary antibodies were detected following incubation for $2 \mathrm{~h}$ with horseradish peroxidase (HRP)-conjugated secondary antibodies (diluted to 1:5000 in blocking buffer). The detected membranes were incubated for $5 \mathrm{~min}$ with the enhanced chemiluminescence (ECL) detection reagent in the dark and exposed to a sheet of diagnostic X-ray film in a film cassette for $10 \mathrm{~min}$. The relative protein expression was normalized to that of $\beta$-actin, which served as an internal control, using a software (Alpha Innotech, ver. 4.0.0, San Leandro, CA, USA).

\section{Immunofluorescence}

We cultured COCs on sterilized glass coverslips and fixed them with $4 \%$ paraformaldehyde, followed by blocking with $0.1 \%$ BSA in PBS. Dehydration and permeabilization were performed by freezing the slides at $-20{ }^{\circ} \mathrm{C}$ in $5 \mathrm{mM} 0.1 \%$ Triton X-100 in PBS. After blocking with $3 \%$ BSA in PBS, slides were incubated with an antibody against the active form of MAP1LC3A, ATG5, Caspase-3, and 20a-HSD at 1:150 dilutions. The slides were washed and incubated with anti-rabbit and anti-mouse IgG conjugated to Alexa Fluor 488 or Alexa Fluor 594 (Molecular Probes). Nuclei were counterstained with 1 $\mathrm{g} / \mathrm{mL}$ Hoechst 33258, and slides were mounted using fluorescent mounting medium (Dako, Carpinteria, CA). Images were acquired using Olympus AX70 fluorescence microscope fitted with a CCD color camera.

\section{Statistical analyses}

Real-time RT-PCR results were analyzed for statistical significance using the SAS package (Statistical Analysis System, Institute, version 9.4, Cary, NC, USA). Data were subjected to generalized linear model (GLM) of SAS and are shown as mean \pm standard deviation (SD). The significant difference between groups was determined at $\mathrm{p}<0.05$.

\section{RESULTS}

\section{Quality analysis of matured oocytes following treatment}

The results of the quality evaluation of in vitro matured oocytes cultured in different treatment media are shown in Figure 1. For the oocytes matured at $44 \mathrm{~h}$, the proliferation rate of cumulus cells was higher in the presence of FSH and FSH + LH than that observed in untreated controls and LH-treated cells. Rapamycin supplementation resulted in an increase in the proliferation of cumulus cells in the control and FSH treatment groups; however, the proliferation rate decreased in $\mathrm{LH}$ and $\mathrm{FSH}+\mathrm{LH}$ treatment groups. In particular, the necrosis rate was higher in untreated control, rapamycin $+\mathrm{LH}$, and rapamycin + FSH + LH groups than the other groups (Table 3).

\section{Expression of autophagic and apoptotic mRNAs in matured oocytes}

The evaluation of the expression of apoptosis or autophagyrelated genes revealed that the expressions of these mRNAs were different in each treatment group (Figure 2). Real-time PCR analysis results showed that the expression of autophagyassociated genes (MAP1LC3A and ATG5) was higher in FSH and rapamycin treatment groups, while their expressions were 
Table 3. Oocytes stage of in-vitro matured cultured for $44 \mathrm{~h}$ in each treatment medium

\begin{tabular}{|c|c|c|c|c|c|c|c|c|c|}
\hline \multicolumn{10}{|c|}{ No. of maturation of oocytes (MII) } \\
\hline & \multirow{2}{*}{ No. of oocyte } & \multicolumn{2}{|c|}{ Control } & \multicolumn{2}{|c|}{ FSH } & \multicolumn{2}{|c|}{ LH } & \multicolumn{2}{|c|}{$\mathrm{FSH}+\mathrm{LH}$} \\
\hline & & MI & MII & MI & MII & MI & MII & MI & MII \\
\hline \multirow{2}{*}{ Non-rapamycin } & \multirow{4}{*}{$\begin{array}{c}\text { Each } \\
200\end{array}$} & \multicolumn{2}{|c|}{$15 \%$} & \multicolumn{2}{|c|}{$10 \%$} & \multicolumn{2}{|c|}{$5 \%$} & \multicolumn{2}{|c|}{$1.5 \%$} \\
\hline & & \multicolumn{2}{|c|}{$43 \%$} & \multicolumn{2}{|c|}{$70 \%$} & \multicolumn{2}{|c|}{$40 \%$} & \multicolumn{2}{|c|}{$60 \%$} \\
\hline \multirow{2}{*}{ Rapamycin-Supplement } & & \multicolumn{2}{|c|}{$20 \%$} & \multicolumn{2}{|c|}{$12.5 \%$} & \multicolumn{2}{|c|}{$2.5 \%$} & \multicolumn{2}{|c|}{$25 \%$} \\
\hline & & \multicolumn{2}{|c|}{$75 \%$} & & & \multicolumn{2}{|c|}{$35 \%$} & \multicolumn{2}{|c|}{$37.5 \%$} \\
\hline
\end{tabular}
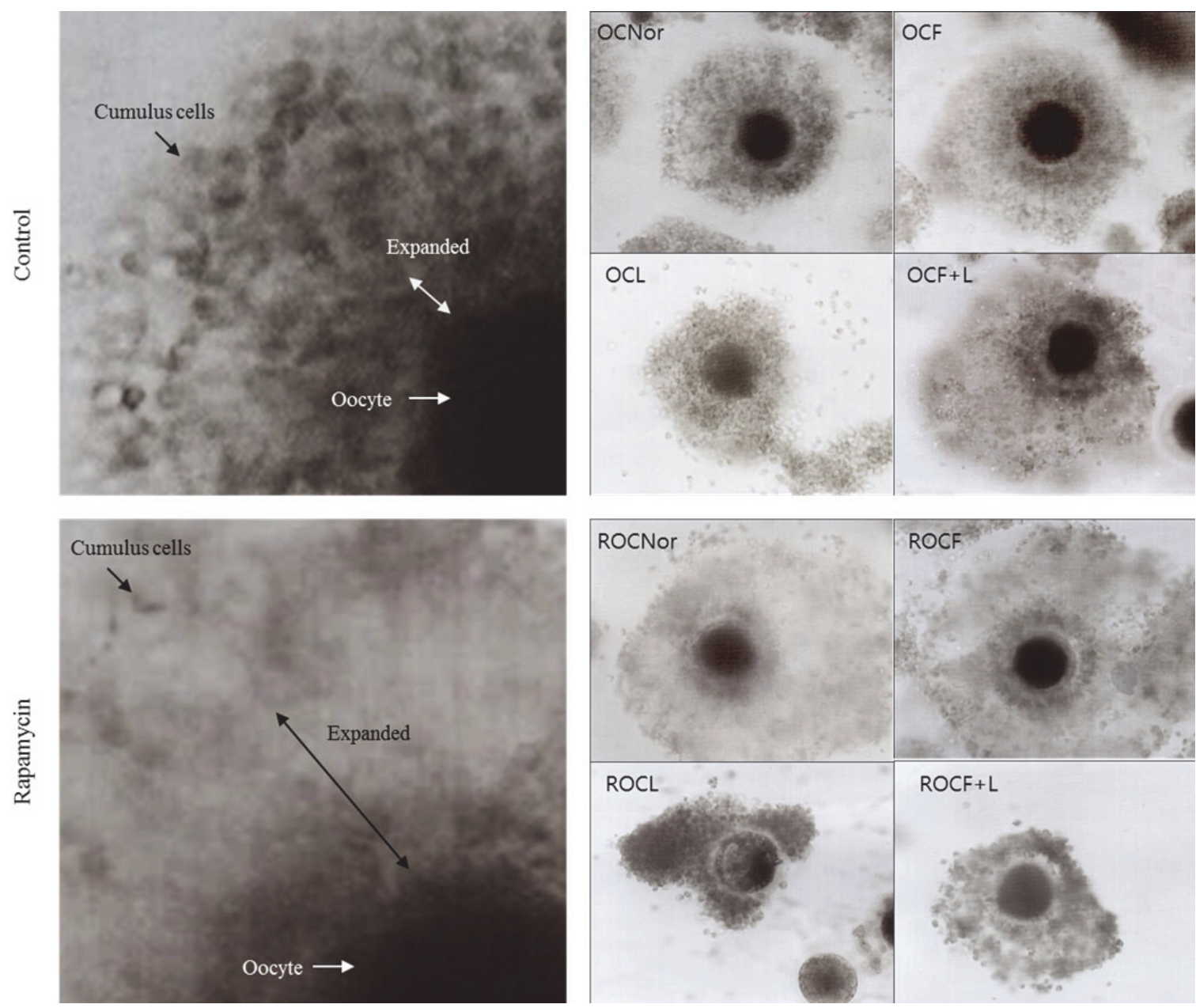

Figure 1. Maturation quality of pig cumulus-oocyte complexes. 200' magnification. OCNor): non-treated oocyte; OCF): FSH-treated oocyte; OCL): LH-treated oocyte; OCF+L): FSH + LH-treated oocyte; ROCNor): rapamycin-supplemented oocyte; ROCF): rapamycin + FSH-treated oocyte; ROCL): rapamycin + LH-treated oocyte; ROCF+L): rapamycin + FSH + LH-treated oocyte.

low in non-rapamycin treatment groups and in the presence of LH. In addition, the expression of anti-progesterone gene (20a -HSD) was higher in the matured oocytes from the non-rapamycin treatment groups than in oocytes from rapamycin treatment groups. However, 20a-HSD gene expression decreased upon treatment with FSH + LH. The expression of caspase-3 mRNA increased in FSH + LH treatment group (OCFL), while the levels of caspase-3 mRNA were similar between rapamycin treatment groups and untreated controls. Thus, the expressions of autophagy and apoptosis-associated genes (MAP1LC3A, ATG5, and caspase-3) were strongly induced by LH in rapamycin media, while the mRNA levels of these genes were lower in the control groups than in treatment groups. On the other hand, the expression of anti-progesterone protein (20a-HSD) was higher in the control group and strongly induced by treatment with FSH than with LH. 

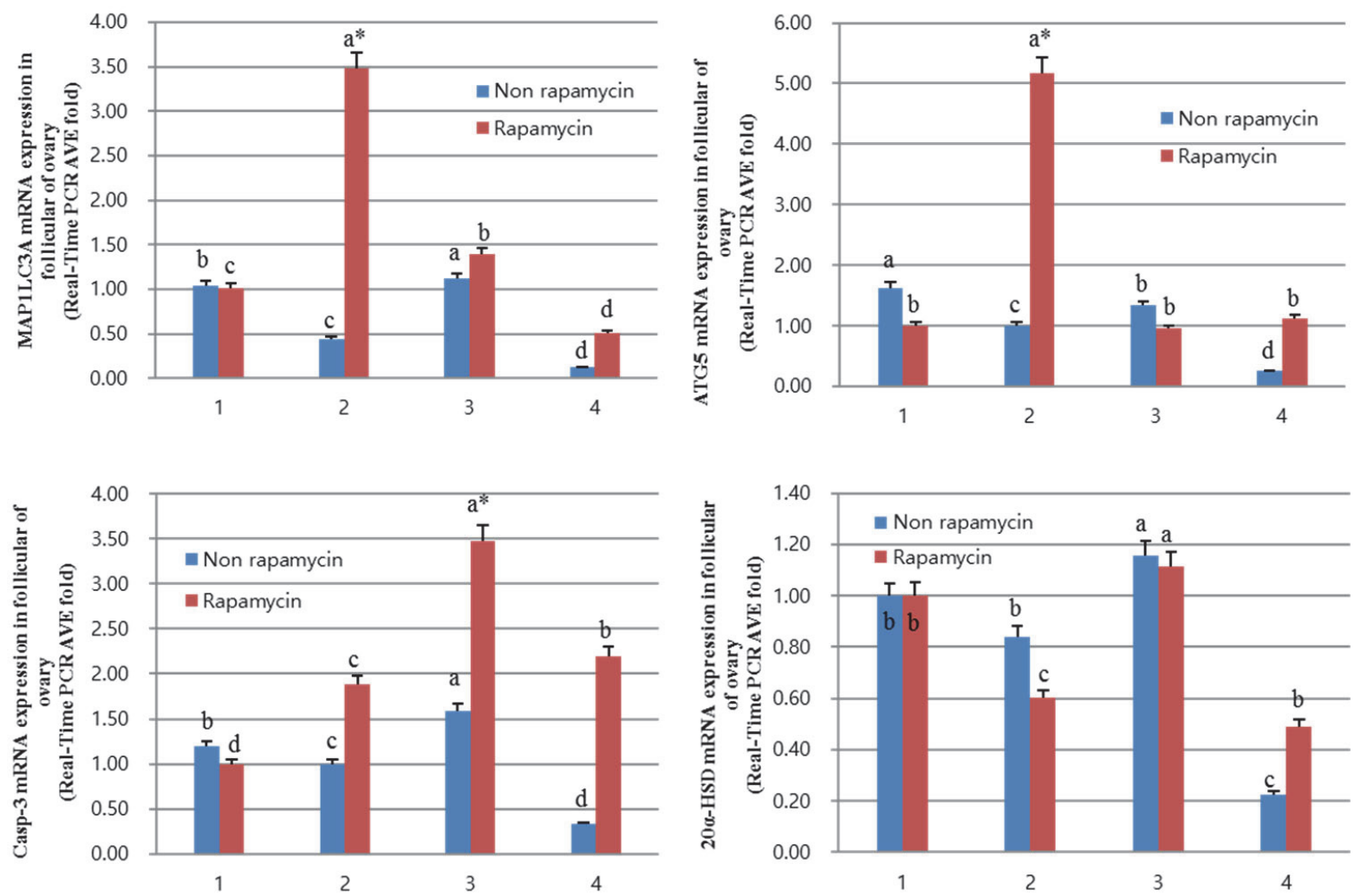

Figure 2. Expression analysis of autophagy- and apoptosis-related genes in cumulus-oocyte complexes of pig. 1) Control; 2) FSH treatment; 3) $\mathrm{LH}$ treatment; 4) FSH + LH treatment. ${ }^{a, b, c, d}$ Differentletterswithinthesamecolumnrepresentasignificantdifference( $(p, 0.05)$.

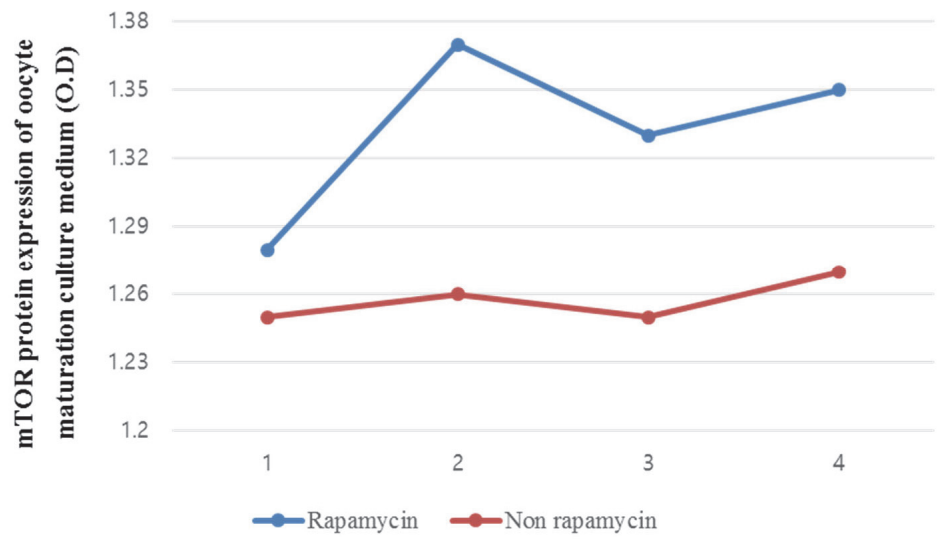

Figure 3. ELISA assay for mTOR expression in matured oocytes. 1) Control; 2) FSH treatment; 3) LH treatment; 4) FSH + LH treatment.

\section{Expression of survival-related genes}

The results of ELISA assay for mTOR protein are shown in Figure 3. The concentrations (optical density [O.D.] values) of $\mathrm{mTOR}$ in the rapamycin-treated groups were $1.28 \pm 0.120,1.37$ $\pm 0.153,1.33 \pm 0.143$, and $1.35 \pm 0.145$ for control, FSH, LH, and FSH-LH treatment, respectively, and tended to be higher following FSH treatment; however, the concentrations of mTOR were low in non-rapamycin treatment groups. The values of
mTOR in non-rapamycin treatment groups were $1.25 \pm 0.023$, $1.26 \pm 0.033,1.25 \pm 0.10$, and $1.27 \pm 0.123$ for control, FSH, LH, and FSH + LH groups, respectively.

\section{Detection of proteins related to programmed cell death $(P C D)$ in matured oocytes}

We compared the levels of autophagic and apoptotic proteins and found that the expressions of MAP1LC3A and ATG5 


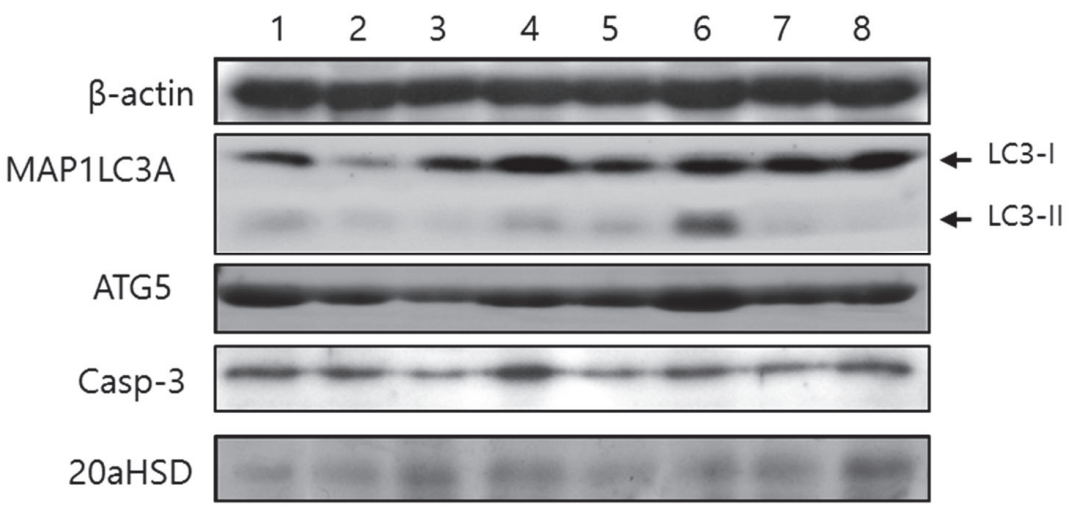

Figure 4. Immunoblot analysis of 20a-HSD, caspase-3, ATG5, and MAP1LC3A proteins in cumulus-oocyte complexes (COCs). Lane 1: Control, Lane 2: FSH, Lane 3: LH, Lane 4: FSH + LH, Lanes 5 to 8: Rapamycin supplementation.

proteins were higher in matured oocytes from the rapamycin treatment groups than in oocytes from the normal group, irrespective of the hormonal treatment. In contrast, the level of caspase-3 protein was higher in matured oocytes from nonrapamycin treatment groups (Figure 4).

The expression levels of MAP1LC3A and ATG5 proteins in rapamycin treatment group were relatively higher with FSH treatment than with $\mathrm{LH}$ treatment. However, these proteins were expressed at low levels in all non-rapamycin control media groups. On the other hand, the level of caspase- 3 protein was higher in the normal (OCN), FSH (OCF), and FSH + LH (OCFL) treatment groups than in LH treatment group (OCL). Overall, the expression of autophagic proteins (MAP1LC3A and ATG5) was more readily induced as compared with the expression of apoptotic proteins following rapamycin supplementation. The expression of 20a-HSD in LH and FSH + LH treatment groups was mostly higher than that in other groups.

\section{Immuno-detection of autophagic and apoptotic proteins in matured oocytes}

Immunofluorescence detection of matured oocytes from each treatment medium was performed to determine the population that contributed to the expression of autophagic and apoptotic proteins (Figure 5 and 6). Levels of MAP1LC3A and ATG5 proteins were elevated in matured oocytes from the rapamycin treatment group (ROCN or/and ROCF) as compared to control groups (Figure 3). On the other hand, the expression of caspase- 3 remained low in all rapamycin treatment groups except $\mathrm{FSH}+\mathrm{LH}$ treatment group. Matured oocytes from non-rapamycin treatment group showed higher levels of caspase-3 and lower levels of MAP1LC3A in all conditions. However, 20a-HSD expression was low in the rapamycin treatment groups and high in non-rapamycin treatment groups (OCL and OCFL).

The results from western blot analyses showed that the expression of autophagy-associated proteins (MAP1LC3A and ATG5) in the rapamycin treatment groups was higher following treatment with FSH (ROCF) than with LH. For the non-rapamycin treatment groups, the expression was higher with $\mathrm{FSH}+\mathrm{LH}$ than with FSH or LH. The apoptotic gene (caspase-3 and 20a -HSD) was more strongly expressed in the oocytes of nonrapamycin treatment groups in the presence of FSH and FSH + LH than with LH. The apoptotic gene expression was high in all non-rapamycin groups and the lowest expression was reported for the rapamycin groups. Taken together, these results suggest that PCD type II pathway (autophagy) is prominent in rapamycin and FSH treatment groups, while type I pathway (apoptosis) is prominent in the non-rapamycin and LH and/or FSH + LH groups.

\section{DISCUSSION}

Proper maturation of oocytes is important for the production of high-quality blastocysts during IVF. Unfortunately, only a small percentage of immature oocytes from the original pool proceeds to the blastocyst stage during IVM, contributing to pregnancy. The best described PCD types are apoptosis and autophagy, which play a major role in both embryonic development and aging; however, the regulatory mechanisms underlying oocyte maturation are questionable (Klionsky et al., 2007), although recent reports have demonstrated the importance of proper execution of PCD in this process.

Several studies have shown that cytokinesis is an essential step during meiosis for the asymmetric division to allow retention of 


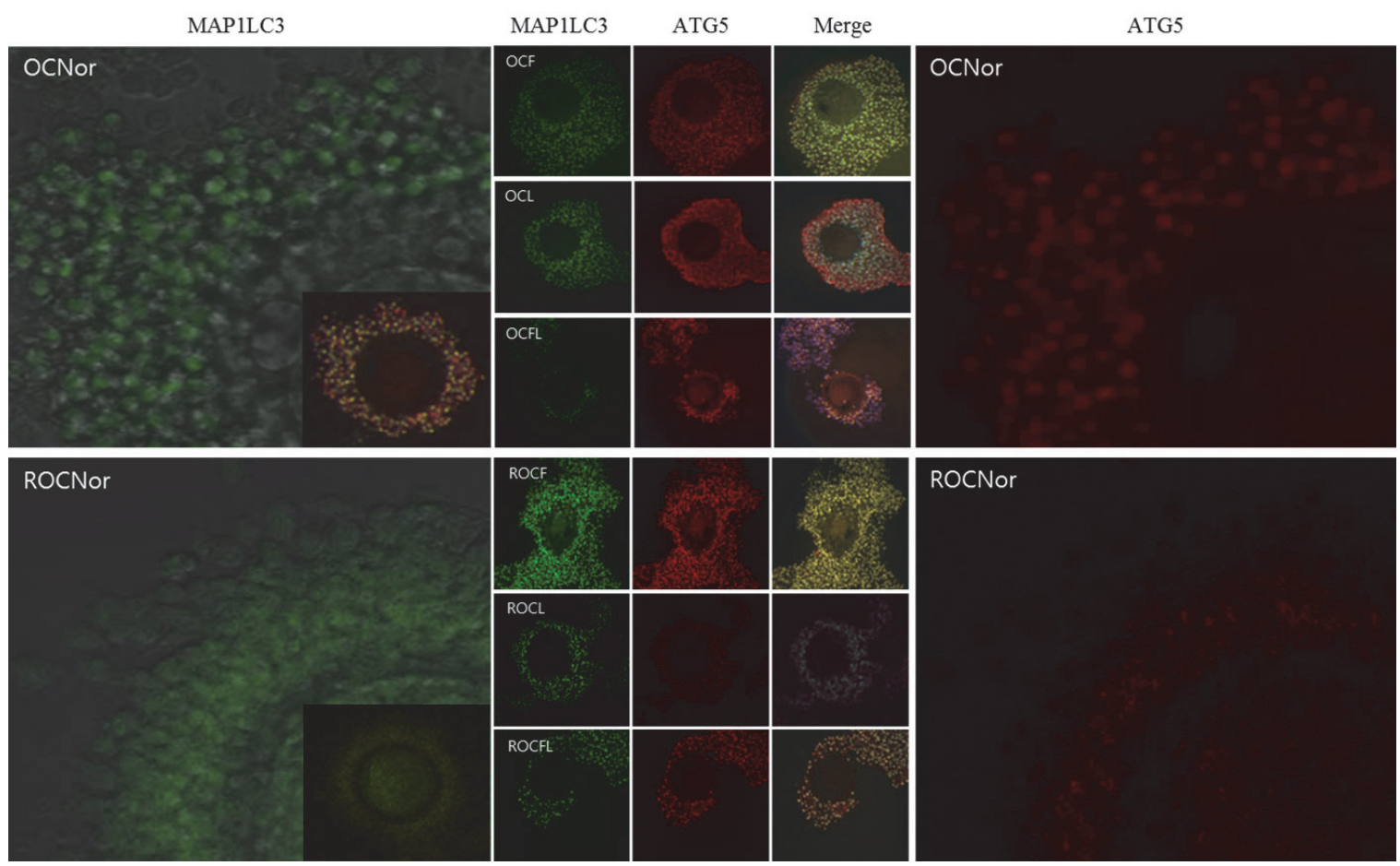

Figure 5. Immunofluorescence analysis of autophagy-related proteins in oocytes. Oocytes were maturated in the absence or presence of hormones and rapamycin. OCNor): non-treated oocyte; OCF): FSH-treated oocyte; OCL): LH-treated oocyte; OCF+L): FSH + LH-treated oocyte; ROCNor): rapamycin-supplemented oocyte; ROCF): rapamycin + FSH-treated oocyte; ROCL): rapamycin + LH-treated oocyte; ROCF $+\mathrm{L}$ ): rapamycin + FSH + LH-treated oocyte.

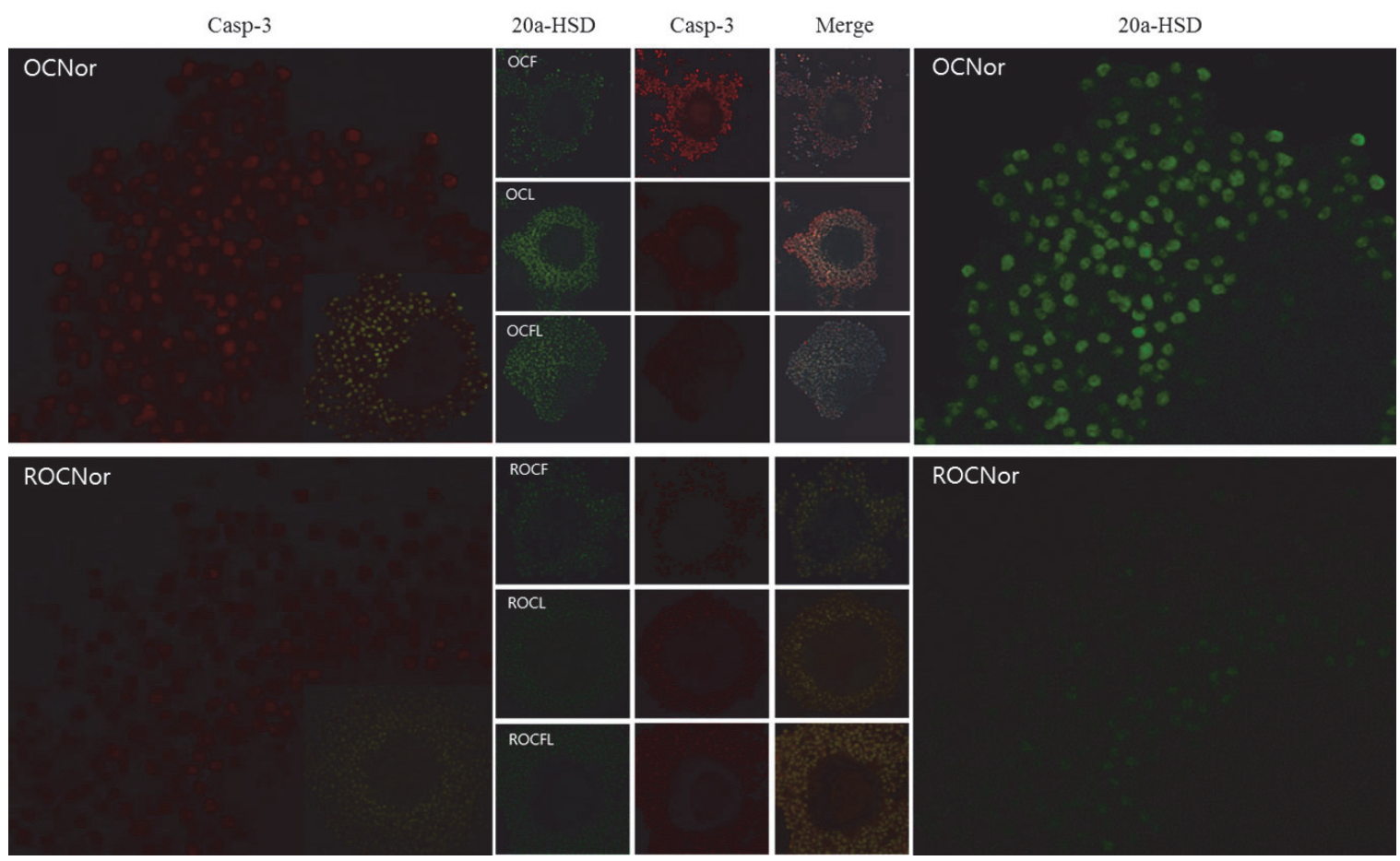

Figure 6. Immunofluorescence analysis of apoptosis-related proteins in oocytes. Oocytes were maturated in the absence or presence of hormones and rapamycin. OCNor): non-treated oocyte; OCF): FSH-treated oocyte; OCL): LH-treated oocyte; OCF+L): FSH + LH-treated oocyte; ROCNor): rapamycin-supplemented oocyte; ROCF): rapamycin + FSH-treated oocyte; ROCL): rapamycin + LH-treated oocyte; ROCF+L): rapamycin + FSH + LH-treated oocyte. 
maternal components required for the early development (Maro et al., 2002). In particular, autophagy activation has been observed in immature oocytes (Bang et al., 2014). Rapamycin treatment induced autophagy, thereby improving IVM of porcine oocytes (Song et al., 2014). Our previous work shows that hormones and rapamycin affected the follicular maturation in porcine. Based on this observation, we assumed that rapamycin and hormone treatment may exhibit positive effects on porcine oocyte maturation. We found that IVM rate was higher for oocytes cultivated in rapamycin media than those cultured in the normal medium. The treatment with rapamycin, an autophagy activation factor, increased cumulus cell expansion and cytoplasm maturation in untreated control and FSH-treated groups but decreased the maturation rate in LH-treated group. On the other hand, the maturation rate was greatly decreased in $\mathrm{FSH}+\mathrm{LH}$ treatment group. These observed results are different from those established in a previous report on IVM of oocytes in the presence of FSH + LH hormones (Manabe et al., 2004). The expression of proteins related to apoptosis suggests that PCD type II pathway (autophagy) is prominent in rapamycin and FSH hormone treatment groups, while the type I pathway (apoptosis) is dominant in the non-rapamycin and LH treatment groups. The differential response is probably associated with the functional difference of these hormones, as FSH contributes to the maturation of follicles by accelerating the development of granulosa cells, while LH remodels granulosa cells into luteal cells (Song et al., 2014; Wei et al., 2017). Thus, autophagy is preferred over apoptosis during the oocyte maturation in the presence of rapamycin. The main role of PCD in oocytes is to eliminate the poor-quality oocytes (Lobascio et al., 2007). In addition, the proliferation rates of cumulus cells increased from COCs treated with rapamycin to those treated with $\mathrm{FSH}$, while it decreased from non-rapamycin treatment groups to $\mathrm{FSH}+\mathrm{LH}$ treatment group. These results indicate that autophagy is preferred over apoptosis during the development of cumulus cells and oocytes (Cuervo, 2004; Lobascio et al., 2007). Apoptosis or anti-progesterone regulates the maturation of oocytes, as evident from the high expression of 20a-HSD, which is known to activate apoptosis, in a previous study (De Felici et al., 2005). During oocyte maturation, cell death mostly occurs through apoptosis (De Felici et al., 2005). However, the causes and molecular mechanisms underlying the process of oocyte death remain unclear (Morita and Tilly, 1999; Tilly, 2001; De Felici et al., 2005). Although we failed to demonstrate the direct control of PCD by rapamycin and hormones investigated in this study, our results clearly indicate that maturation of oocytes is differentially regulated in treatment media. In addition, the type II pathway of PCD is preferred to obtain high-quality matured oocytes, while the type I PCD involved in apoptosis is the main contributor for the poor-quality oocytes in porcine. Thus, apoptosis is very important for the understanding of the functional changes during IVM of oocytes and to illuminate the autophagy formation mechanism through the interaction with hormones. This observation may be used as a measure for the general solution in animal and human IVF problems.

\section{ACKNOWLEDGMENTS}

This work was supported by a research grant from Hankyong National University for a academic exchange program in 2016.

\section{REFERENCES}

Bang S, Shin H, Song H, Suh CS, Lim HJ. 2014. Autophagic activation in vitrified-warmed mouse oocytes. Reproduction. 148:11-19.

Brackett BG, Bousquet D, Boice ML, Donawick WJ, Evans JF, Dressel MA. 1982. Normal development following in vitro fertilization in the cow. Biol Reprod. 27(1):147-158.

Cohen J, Mandelbaum J, Plachot M. 1980. Maturation and in vitro fertilisation of human oocytes recovered after spontaneous ovulation or after induction of ovulation by use of gonadotrophins (preliminary results) (author's transl). J Gynecol Obstet Biol Reprod (Paris).9(5):523-530.

Cuervo AM. 2004. Autophagy: many paths to the same end. Mol Cell Biochem. 263:355-372.

De Felici M, Klinger FG, Farini D, Scaldaferri ML, Iona S \& Lobascio M. 2005. Establishment of oocyte population in the fetal ovary: primordial germ cell proliferation and oocyte programmed cell death. Reproductive Biomedicine Online. $10182-191$.

Edwards MJ. 1969. Congenital defects in guinea pigs: prenatal retardation of brain growth of guinea pigs following hyperthermia during gestation.Teratology. 2(4):329-336.

Eppig JJ, O'Brien M, Wigglesworth K. 1996. Mammalian oocyte growth and development in vitro. Mol Reprod Dev. 44(2):260-273.

Eppig JJ. 1996. Coordination of nuclear and cytoplasmic oocyte maturation in eutherian mammals. Reprod Fertil Dev. 8(4):485-489. 
Eyestone WH1, First NL. 1989. Co-culture of early cattle embryos to the blastocyst stage with oviducal tissue or in conditioned medium. J Reprod Fertil. 85(2):715-720.

Kikuchi K, Nagai T, Ding J, Yamauchi N, Noguchi J, Izaike Y. 1999. Cytoplasmic maturation for activation of pig follicular oocytes cultured and arrested at metaphase I.J Reprod Fertil. 116(1):143-156.

Klionsky DJ, Cuervo AM, Dunn WA Jr. 2007. How shall I eat thee? Autophagy. 3:413 - 416 .

Lobascio AM, Klinger FG, Scaldaferri ML, Farini D and De Felici M. 2007. Analysis of programmed cell death in mouse fetal oocytes. Reproduction. $134241-252$.

Manabe N, Goto Y, Matsuda-Minehata F, Inoue N, Maeda A, Sakamaki K, Miyano T. 2004. Regulation mechanism of selective atresia in porcine follicles: regulation of granulosa cell apoptosis during atresia. J Reprod Dev. 50(5): 493-514.

Maro B, Verlhac MH. 2002. Polar body formation: new rules for asymmetric divisions. Nat Cell Biol. 4: 281-283.

Morita Y \& Tilly JL. 1999. Oocytes apoptosis: like sand through an hourglass. Developmental. Biology. 213: 1 - 17.
Singh B, Meng L, Rutledge JM, Armstrong DT. 1997. Effects of epidermal growth factor and follicle-stimulating hormone during in vitro maturation on cytoplasmic maturation of porcine oocytes. Mol Reprod Dev. 46(3): 401-407.

Song BS, Kim JS, Kim YH, Sim BW, Yoon SB, Cha JJ, Choi SA, Yang HJ, Mun SE, Park YH. 2014. Induction of autophagy during in vitro maturation improves the nuclear and cytoplasmic maturation of porcine oocytes. Reprod Fertil Dev. 26:974-981.

Tilly JL. 2001. Commuting the death sentence: how oocytes strive to survive. Nature. 2: $838-848$.

Wei S, Shen X, Gong Z, Deng Y, Lai L, Liang H. 2017. FSHR and LHR Expression and Signaling as Well as Maturation and Apoptosis of Cumulus-Oocyte Complexes Following Treatment with FSH Receptor Binding Inhibitor in Sheep. Cell Physiol Biochem. 22;43(2):660-669.

Yoshida M, Cran DG, Pursel VG. 1993. Confocal and fluorescence microscopic study using lectins of the distribution of cortical granules during the maturation and fertilization of pig oocytes. Mol Reprod Dev. 36(4):462-468. 